\title{
Acute graft-versus-host disease: inflammation run amok?
}

\author{
Joseph H. Antin \\ Department of Adult Oncology, Dana-Farber Cancer Institute; and Department of Medicine, Brigham and Women's Hospital; \\ Harvard Medical School, Boston, Massachusetts, USA
}

Address correspondence to: Joseph H. Antin, Dana-Farber Cancer Institute, 44 Binney Street, Boston, Massachusetts 02115, USA. Phone: (617) 632-2525; Fax: (617) 632-5175; E-mail: jantin@partners.org.

Hematopoietic stem cell transplantation (HSCT) has substantially fulfilled its promise as a curative therapy for hematopoietic disorders and some disorders of metabolism. More recently, it has been investigated for its potential in establishing tolerance for organ transplantation. In its typical application, conditioning steps involving cytotoxic radiation or chemotherapy are administered first, after which some combination of stem cells, lymphocytes, dendritic cells, and other cells is infused into the recipient. In the treatment of malignant disorders, the goal is to eradicate the cancer through a combination of cytotoxic injury and destruction of the malignancy by immunologic attack, a response described as the graft-versusleukemia (GVL) effect. This approach is limited by (a) collateral tissue damage from the conditioning regimen, (b) immunological injury to normal tissues (graft-versus-host disease [GVHD]), and (c) the ability of the malignancy to survive this dual assault.

\section{Barriers to GVHD}

Decades of basic and clinical research have demonstrated that $\mathrm{T}$ cells are the principal orchestrators of both GVHD and GVL, but the mechanisms of GVHD induction are not yet fully elucidated. In clinical transplantation using histocompatible donors, it is thought that $\mathrm{T}$ cells recognize minor histocompatibility antigens presented by MHC class I and class II molecules. This recognition results in activation and proliferation of the $\mathrm{T}$ cells followed by cytotoxic attack on target organs and the resultant clinical manifestations of GVHD. It is well recognized that GVHD primarily involves skin, gut, liver, and perhaps lung (1). Despite the susceptibility of kidney and heart to allograft rejection, these organs are not targets of GVHD. Pathologically, there is a modest inflammatory infiltrate but substantial damage to the basilar layer of the skin, the intestinal crypts, and the portal area of the liver. Apoptosis of cells in these areas results in rash, mucosal denudation and subsequent diarrhea, and/or biliary stasis. In severe cases it can be fatal. One hint that helps explain the organ selectivity and some aspects of the pathophysiology of GVHD is the target organ distribution per se - these organs are all primary or secondary barriers to the environment. The functions of skin and gut are selfevident, and the liver's extensive reticuloendothelial system reinforces the barrier function of the endotoxin-rich intestinal mucosa.

While we refer to the GVH reaction as a "disease," it is better considered as a normal reaction of normal, donor lymphocytes to what appears to the immune system to be a serious infection. Thus, a reasonable framework in which to consider the induction of GVHD is that there is damage to the epithelium and endothelium by the conditioning regimen itself as well as by effects of the underlying disease. The injured tissues respond as if there were a serious infection, with the production of factors - cytokines, chemokines, and adhesion molecules, among others that signal the immune system that infection or injury has occurred. The damaged tissues express antigens that are perceived as foreign by the immune system and are considered targets for elimination. The donor lymphocytes are in a milieu that fosters their direct attack on epithelia, causing tissue damage through perforin, granzyme $\mathrm{B}$, and/or Fas/FasL interactions. They may also recruit other effector cells, such as granulocytes and macrophages (partially summarized in Figure 1). In addition, the dysregulated production of inflammatory cytokines, such as TNF$\alpha$, IFN- $\gamma$, IL- 1 , and others, may cause direct tissue damage. Endotoxin may leak through these injured barriers, where it tends to fuel the response. Further injury to the barrier results in more release of endotoxin and exacerbation of the inflammation. Indirect support

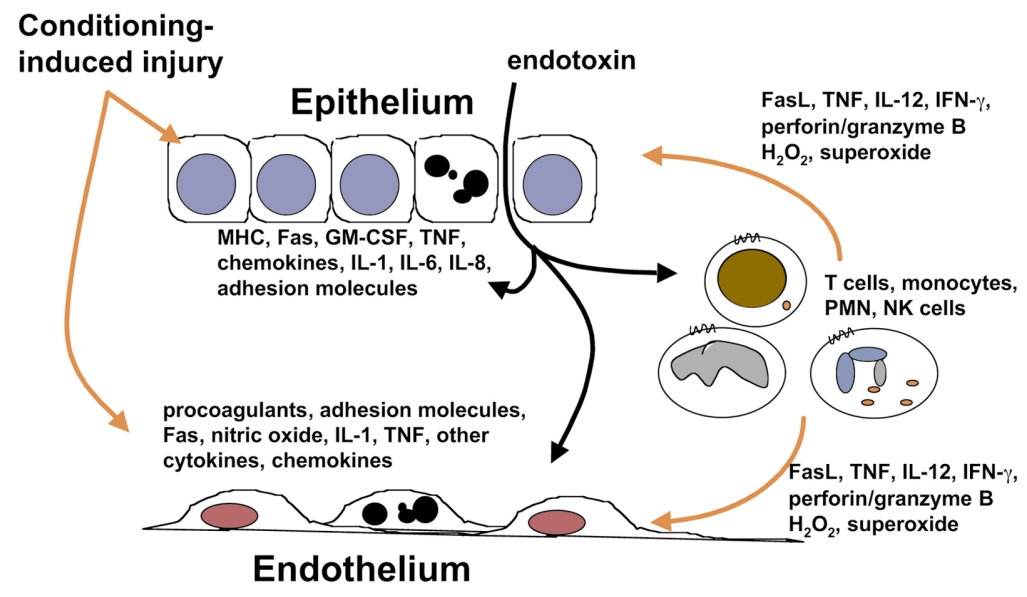

Figure 1

Pathophysiology of GVHD. Injury to host epithelia and endothelium generates injury signals that recruit donor T cells. In this inflammatory milieu, the donor T cells recognize minor histocompatibility antigens, resulting in activation and proliferation. The T cells then cause further injury through both specific and nonspecific mechanisms. This process is amplified by endotoxin. The clinical manifestations of this process are seen in GVHD. PMN, polymorphonuclear leukocyte; NK, natural killer. 
for this concept can be found in experimental transplantation and in some clinical settings where transplantation in low bacteria environments strikingly limits the risk of GVHD $(2,3)$.

In this context, the report by Kuroiwa and colleagues in a recent issue of the JCI provides indirect evidence substantiating the importance of mucosal integrity in preventing GVHD (4). By repeatedly transfecting a human $\mathrm{HGF}$ cDNA into skeletal muscle, they were able to maintain continuous levels of the polyfunctional cytokine during transplantation. They observed that two of the characteristic findings in GVHD, crypt cell apoptosis and infiltration of lymphocytes into the portal triad, were absent in treated animals. Expression of TNF- $\alpha$ and IFN- $\gamma$ was also reduced, and IL-12 levels were low. HGF did not appear to have direct effects on $\mathrm{T}$ cell proliferation, cytotoxicity, or migration. It seems more likely that by abrogating intestinal apoptosis, this treatment limited endotoxin-mediated tissue damage and thus interrupted the inflammatory cascade. There may also be beneficial effects on the liver that limited lymphocyte infiltration.

Cooke and colleagues, writing in the current issue of the JCI, took a more direct approach to blocking GVHD pathogenesis (5). These investigators show that B975, a direct competitive antagonist of endotoxin, attenuates the inflammatory response following transplantation and allows for substantial, although incomplete, improvements in GVHD score and survival. Cooke et al. treated the animals in the first 6 days after HSCT, when the donor $\mathrm{T}$ cells are probably maximally stimulated by the host injury, and observed a concomitant reduction in inflammatory cytokine levels. Furthermore, unlike most other approaches that have been tested to control GVHD, this treatment is not associated with a parallel suppression of the beneficial GVL effect. This welcome finding implies that GVL is largely mediated through direct $\mathrm{T}$ cell cytotoxicity rather than the more nonspecific effects described above.

\section{Future refinements \\ of GVHD therapy}

It is clear that GVHD is a complex process that is unlikely to be controllable with a single agent. Very encouraging studies of single-cytokine inhibition in mouse models have not translated well to human trials. Most studies show that partial benefits can occur through a variety of approaches, including T cell depletion, immunophilin inhibition, cytokine inhibition, manipulation of costimulation, and the use of antiproliferative agents, but these interventions are rarely used synergistically. A useful strategy will be to attempt to control GVHD by recognizing the underlying pathophysiology and interfering with disparate steps along the pathway; for instance, maintaining gut integrity, preventing cytokine upregulation by endotoxin, and interfering with $T$ cell activation. If interventions are chosen wisely, it may be possible to control the inflammatory aspect of GVHD while sparing the critically important antileukemic effect.

1. Glucksberg, H., et al. 1974. Clinical manifestations of graft-versus-host disease in recipients of marrow from HLA-matched sibling donors. Transplantation. 18:295-304.

2. Storb, R., et al. 1983. Graft-versus-host disease and survival in patients with aplastic anemia treated by marrow grafts from HLA-identical siblings. Beneficial effects of a protective environment. N. Engl. J. Med. 308:302-307.

3. Beelen, D.W., et al. 1992. Evidence that sustained growth suppression of intestinal anaerobic bacteria reduces the risk of acute graft-versus-host disease after sibling marrow transplantation. Blood. 80:2668-2676.

4. Kuroiwa, T., et al. 2001. Hepatocyte growth factor ameliorates acute graft-versus-host disease and promotes hematopoietic function. J. Clin. Invest. 107:1365-1374.

5. Cooke, K.R., et al. 2001. LPS antagonism reduces graft-versus-host disease and preserves graft-versus-leukemia activity after experimental bone marrow transplantation. J. Clin. Invest. 107:1581-1589. 